\title{
Use of Metabolomics as a Diagnostic Tool of Urosepsis in Adult Patients
}

\author{
Manodeep Sen*, Tanushri Chatterji \\ *Department of Microbiology, Dr. Ram Manohar Lohia Institute of Medical Sciences (RMLIMS), \\ Vibhuti Khand, Gomti Nagar, Lucknow
}

Keywords: Urosepsis, metabolic profile, NMR spectroscopy.

Urosepsis is a combined term defining sepsis caused by the urinary tract infection (UTI)(Book, Lehmann et al. 2005 ; Mikkelsen, Miltiades et al. 2009). Urosepsis is one of the leading cause of mortality globally (Dellinger, Levy et al. 2008). The prompt and accurate diagnosis is quite time consuming and often results to false positive cases. Hence, to overcome the limitations we have approached the use of metabolomics for metabolomic profiling in serum and urine samples.

The editors have published a pilot study on rapid and efficient diagnosis of urosepsis using the technique of ${ }^{1} \mathrm{H}$ NMR spectroscopy(Singh, Chatterji et al. 2016).

The study design was broadly classified into two datasets i.e. urosepsis cases and healthy controls. On the basis of clinic-pathological parameters urosepsis cases were defined as positive urine culture with serum PCT cut off levels (>0.5 ng/ml) and /or positive blood culture for bacterial pathogens(Wilson and Gaido 2004). Clinical signs and symptoms of the cases were found to be statistically significant, which included evidence of fever, high respiratory and high heart rate and significant cell count ( $>5$ WBC/HPF)(Stamm 1983).

1D NMR experiments were performed on collected serum and urine samples, followed by the identification of 34 and 35 metabolites respectively. The metabolites were characterized and assigned as reported in literature (Martin, Sprenger et al. 2009)and were compared with the standard NMR spectra of metabolites available in the biological magnetic resonance bank (BMRB, www.bmrb.wisc.edu), Human Metabolome Data Base (HMDB, www.hmdb.ca) and through NMR suite 8.1 (Chenomx) software.

Quantification of serum and urine metabolites were further evaluated through multivariate Discriminant Function Analysis (DFA) afforded 93.9\% and 91.7\% correct classification respectively, along with identification of malonate and urea as potential biomarkers for diagnosis of urosepsis from the control group.

Therefore, this study revealed the perspective of metabolomics in conjunction with serum PCT levels as diagnostic tool for early and precise diagnosis.

\section{ACKNOWLEDGEMENTS}

The authors are thankful to Dr. Ram Manohar Lohia Institute of Medical Sciences for intramural funding (IEC 18/12) to carry out the study and Centre of Biomedical Research, Lucknow where the ${ }^{1} \mathrm{H}$ NMR spectroscopy was conducted under the guidance of Prof. Raja Roy.

\section{REFERENCES}

Singh, S., T. Chatterji, et al. (2016). "Serum procalcitonin levels in combination with $1 \mathrm{H}$ NMR spectroscopy: A rapid indicator for differentiation of urosepsis." ClinicaChimiaActa 453: 205214.

Book, M., L. E. Lehmann, et al. (2005 ). "Urosepsis. Current therapy and diagnosis. ." Urologe A. 44(4): 413-22. 
Dellinger, R. P., M. M. Levy, et al. (2008). "Surviving Sepsis Campaign: International guidelines for management of severe sepsis and septic shock: 2008." Critical Care Medicine 36(1): 296-327.

Martin, F. P., N. Sprenger, et al. (2009). "Panorganismal Gut Microbiome-Host Metabolic Crosstalk." Journal of Proteome Research 8(4): 2090-15.

Mikkelsen, M. E., A. N. Miltiades, et al. (2009). "Serum lactate is associated with mortality in severe sepsis independent of organ failure and shock. " Critical Care Medicine 37(5): 1670-77.

Stamm, W. E. (1983). "Measurement of pyuria and its relation to bacteriuria." American Journal of Physiology 75(1B): 53-8.

Wilson, M. L. and L. Gaido (2004). "Laboratory Diagnosis of Urinary Tract Infections in Adult Patients." Clinical Infectious Disease 38(8): 1150-58. 